\title{
Corrigendum: Network deconvolution as a general method to distinguish direct dependencies in networks
}

\author{
Soheil Feizi, Daniel Marbach, Muriel Médard \& Manolis Kellis \\ Nat. Biotechnol. 31, 726-733 (2013); published online 14 July 2013; corrected after print 7 April 2015
}

In the version of this article initially published, six numbers pertaining to the performance of network deconvolution (ND) relative to other methods were incorrect. The corrected numbers (see table below) show that our method performs better than had been reported.

- Four of these numbers correspond to the improvement of ND relative to other regulatory network inference methods. In all four cases, ND improves upon other methods, but the magnitude of the improvement is larger than was reported (ND is $25-87 \%$ better, instead of the reported $22-75 \%$ better).

- The fifth number corresponds to the discrimination power between interacting and noninteracting residues in protein folding prediction. The discrimination was reported as $15 \%$ (fivefold better than mutual information), but it is in fact 22\% (sevenfold better than mutual information).

- The sixth number corresponds to the correlation coefficient between predicted collaboration strength (based on binary weights deconvolved by ND) and true collaboration strength (computed by Newman's method that uses additional information for each pair of authors about the number of co-authored papers and the number of authors on each of these papers). The reported correlation was 0.76 , when the true correlation is 0.74 .

In each case, the reported performance numbers correspond to scaling by the maximum eigenvalue of the observed network, rather than by the maximum eigenvalue of the direct network (as specified in equation 12 in Supplementary Note 1.6).

\begin{tabular}{lll} 
Regulatory network application & Reported ND performance improvement & Actual ND performance improvement \\
\hline $\begin{array}{l}\text { Average ND improvement over mutual information and corr. in DREAM5 } \\
\text { overall (p. 729, paragraph 1 and Fig. 2 legend) }\end{array}$ & $59 \%$ & $66 \%$ \\
$\begin{array}{l}\text { Average ND improvement over mutual information and corr. in DREAM } \\
\text { in silico (p. 729, paragraph 1) }\end{array}$ & $53 \%$ & $61 \%$ \\
$\begin{array}{l}\text { ND improvement in DREAM5 over ARACNE (p. 729, paragraph 1) } \\
\text { ND improvements in DREAM5 over community network (p. 729 paragraph }\end{array}$ & $75 \%$ & $87 \%$ \\
2 and Fig. 2 legend) & & $25 \%$ \\
\hline $\begin{array}{l}\text { Protein folding application } \\
\text { ND discrimination in protein application (p. 730, Fig. 3b correction }\end{array}$ & $15 \%$ (fivefold improvement) & Actual ND discrimination \\
\hline $\begin{array}{l}\text { explained below) } \\
\text { Social network application }\end{array}$ & Reported correlation with Newman weight & Actual correlation with Newman weight \\
\hline $\begin{array}{l}\text { Correlation coefficient between ND weights and Newman weights (p. 731, } \\
\text { paragraph } 1 \text { and Fig. } 4 \text { legend) }\end{array}$ & $R^{2}=0.76$ & $R^{2}=0.74$
\end{tabular}

For one of these numbers (in the protein-folding application), the correction affects a figure panel (Fig. 3b), for which we provide an updated version. The conclusion remains unchanged, namely that ND leads to a substantial increase in discrimination between interacting and noninteracting pairs, but the magnitude of that improvement is stronger than reported (sevenfold improvement instead of the reported fivefold improvement). We show the old and new figures side by side. The difference in the "Before ND" curves stems from the processing of the MI network by the EVfold authors, which was only used in the original version of the figure (MI was recomputed from the original data in the corrected Fig. $3 \mathrm{~b}$ and for all results reported in our paper).
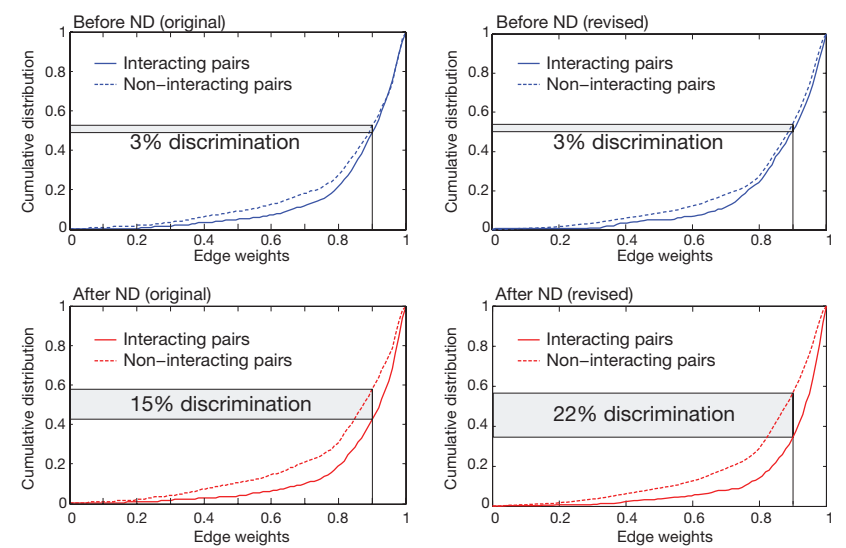

In addition, we have added a panel to Figure 2a to illustrate the robustness of network deconvolution performance in the DREAM application for different eigenvalue scaling parameters, and we have updated the deconvolved networks in our Supplementary Data.

These errors have been corrected in the HTML and PDF versions of the article. 